\title{
Cycloids on the Spherical Surface
}

\author{
Tatiana Olejníková \\ Technical University of Košice, Slovakia \\ Civil Engineering Faculty, Institute of Construction Technology and management \\ e-mail: tatiana.olejnikova@tuke.sk
}

\begin{abstract}
The article describes the creation of the normal cycloidal curve by rotation of the point about the normal of the circle on the spherical surface and binormal cycloidal curve created by rotation of the point about the binormal of the previous cycloidal curve. Cycloidal cyclical surfaces are created by moving circles along the curves lying in the normal plane of the curves. Described cycloidal cyclical surfaces are projected on the spherical surface. Varying parameters of the curves are generated different ornaments on the spherical surface.
\end{abstract}

Key words: cycloidal curve, cycloidal cyclical surface, Frenet-Serret moving trihedron, spherical surface

\section{Introduction}

Let the three-dimensional Euclidean space $\mathrm{E}^{3}$ is determined by the Cartesian coordinate system $(0, x, y, z)$. Let the spherical surface $\Phi$ of radius $r$ and centre in the origin 0 is determined by the parametrical equations

$$
\begin{aligned}
& x=r \cos u \cos v \\
& y=r \cos u \sin v, u \in\langle-\pi / 2, \pi / 2,\rangle, v \in\langle 0,2 \pi\rangle . \\
& z=r \sin u
\end{aligned}
$$

Let $c$ is the circle on this spherical surface determined by the vector function

$$
\mathbf{r}(v)=(r \cos v, r \sin v, 0,1), v \in\langle 0,2 \pi\rangle \text {. }
$$

Let $\left(0^{\prime}, n, b, t\right)$ be the Frenet-Serret moving trihedron of the circle $c$, where $n$ is the principle normal, $b$ is binormal and $t$ is tangent of the circle $c$ at the point $O^{\prime} \in c$ with the unit vectors in the coordinate system $(0, x, y, z)[1]$ 


$$
\begin{gathered}
\mathbf{t}(v)=\left(t_{x}(v), t_{y}(v), t_{z}(v)\right)=\frac{\mathbf{r}^{\prime}(v)}{\left|\mathbf{r}^{\prime}(v)\right|}=(-\sin v, \cos v, 0), \\
\mathbf{b}(v)=\left(b_{x}(v), b_{y}(v), b_{z}(v)\right)=\frac{\mathbf{r}^{\prime}(v) \times \mathbf{r}^{\prime \prime}(v)}{\left|\mathbf{r}^{\prime}(v) \times \mathbf{r}^{\prime \prime}(v)\right|}=(0,0,1), \\
\mathbf{n}(v)=\left(n_{x}(v), n_{y}(v), n_{z}(v)\right)=\mathbf{b}(v) \times \mathbf{t}(v)=(-\cos v,-\sin v, 0) .
\end{gathered}
$$

Frenet-Serret moving trihedron $\left(0^{\prime}, n, b, t\right)$ is represented by the regular square matrix [2]

$$
\mathbf{M}(v)=\left(\begin{array}{cccc}
n_{x}(v) & n_{y}(v) & n_{z}(v) & 0 \\
b_{x}(v) & b_{y}(v) & b_{z}(v) & 0 \\
t_{x}(v) & t_{y}(v) & t_{z}(v) & 0 \\
0 & 0 & 0 & 1
\end{array}\right)=\left(\begin{array}{cccc}
-\cos v & -\sin v & 0 & 0 \\
0 & 0 & 1 & 0 \\
-\sin v & \cos v & 0 & 0 \\
0 & 0 & 0 & 1
\end{array}\right) .
$$

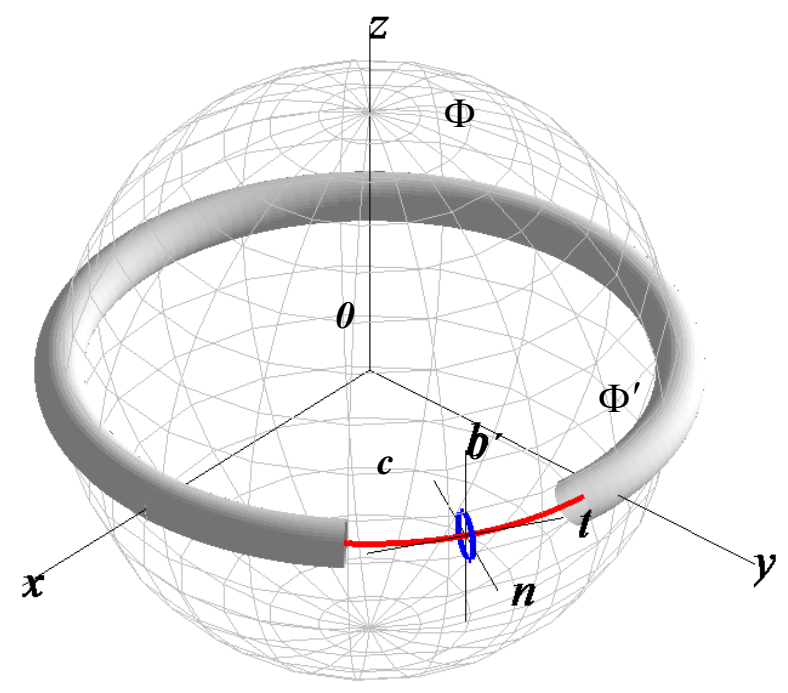

Figure 1: Frenet-Serret moving trihedron $\left(O^{\prime}, n, b, t\right)$ of the circle $c$

In Fig.1 is displayed spherical surface $\Phi$, circle $c$ with Frenet-Serret moving trihedron $\left(O^{\prime}, n, b, t\right)$ and cyclical surface $\Phi^{\prime}$ created by the circle $c^{\prime}=\left(O^{\prime}, r^{\prime}\right)$ moving along the circle $c$ and lying in the normal plane determined by the principal normal $n$ and the binormal $b$ of the circle $c$ at the point $O^{\prime}$.

Then the vector function of the cyclical surface $\Phi^{\prime}$ is

$$
\mathbf{P}^{\prime}(u, v)=\mathbf{r}(v)+\mathbf{c}^{\prime}(u) \cdot \mathbf{M}(v), u \in\langle 0,2 \pi\rangle, v \in\langle 0,2 \pi\rangle,
$$


where $\mathbf{r}(v)$ is vector function of the circle $c$ expressed in equations (2), $\mathbf{M}(v)$ is the transformation matrix of the coordinate system $\left(O^{\prime}, n, b, t\right)$ into the coordinate system $(0, x, y, z)$, (Eq.6) and $\mathbf{c}^{\prime}(u)=\left(r^{\prime} \cos u, r^{\prime} \sin u, 0,1\right), u \in\langle 0,2 \pi\rangle$ is the vector function of the circle $c^{\prime}$.

\section{Creating of the normal cycloidal curve $c_{1}$ of the circle $c$}

Point $\mathrm{P}_{1}\left(0,0, d_{1}, 1\right)$ in the coordinate system $\left(0^{\prime}, n, b, t\right), O^{\prime} \in c$ rotates about the normal $n$ of the circle $c$ with angular velocity $m_{1} v$, the orientation is determined by the parameter $\operatorname{sgn}_{1}$ and creates the cycloidal curve $c_{1}$ which is expressed by vector function

$\mathbf{r}_{\mathbf{1}}(v)=\left(x_{1}, y_{1}, z_{1}, 1\right)=\left(r \cos v-d_{1} \cos m_{1} v \sin v, r \sin v+d_{1} \cos m_{1} v \cos v,-\operatorname{sgn}_{1} d_{1} \sin m_{1} v, 1\right),(8)$

for parameter $v \in\langle 0,2 \pi\rangle$.

Cycloidal cyclical surface $\Phi_{1}^{\prime}$ is created by the movement of the circle $c_{1}^{\prime}=\left(0^{\prime \prime}, r_{1}^{\prime}\right)$ along the cycloidal curve $c_{1}$ and lying in the normal plane determined by the principal normal $n^{\prime}$ and the binormal $b^{\prime}$ of the curve $c_{1}$ at the point $0^{\prime \prime}$.

Let $\left(0^{\prime \prime}, n^{\prime}, b^{\prime}, t^{\prime}\right)$ be the Frenet-Serret moving trihedron of the cycloidal curve $c_{1}$, where $n^{\prime}$ is the principle normal, $b^{\prime}$ is binormal and $t^{\prime}$ is tangent of the curve $c_{1}$ at the point $0^{\prime \prime} \in c_{1}$ with the unit vectors

$$
\begin{gathered}
\mathbf{t}^{\prime}(v)=\left(t_{x}^{\prime}, t_{y}^{\prime}, t_{z}^{\prime}\right)=\frac{\mathbf{r}_{1}(v)^{\prime}}{\left|\mathbf{r}_{1}(v)^{\prime}\right|}, \\
\mathbf{b}^{\prime}(v)=\left(b_{x}^{\prime}, b_{y}^{\prime}, b_{z}^{\prime}\right)=\frac{\mathbf{r}_{1}(v)^{\prime} \times \mathbf{r}_{1}(v)^{\prime \prime}}{\left|\mathbf{r}_{1}(v)^{\prime} \times \mathbf{r}_{1}(v)^{\prime \prime}\right|}, \\
\mathbf{n}^{\prime}(v)=\left(n_{x}^{\prime}, n_{y}^{\prime}, n_{z}^{\prime}\right)=\mathbf{b}^{\prime}(v) \times \mathbf{t}^{\prime}(v) .
\end{gathered}
$$

Matrix

$$
\mathbf{M}_{1}(v)=\left(\begin{array}{cccc}
n_{x}^{\prime}(v) & n_{y}^{\prime}(v) & n_{z}^{\prime}(v) & 0 \\
b_{x}^{\prime}(v) & b_{y}^{\prime}(v) & b_{z}^{\prime}(v) & 0 \\
t_{x}^{\prime}(v) & t_{y}^{\prime}(v) & t_{z}^{\prime}(v) & 0 \\
0 & 0 & 0 & 1
\end{array}\right)
$$


represents the transformation of the coordinate system $\left(0^{\prime \prime}, n^{\prime}, b^{\prime}, t^{\prime}\right)$ (the moving trihedron of the curve $\left.c_{1}\right)$ into the coordinate system $\left(O^{\prime}, n, b, t\right)$ (the moving trihedron of the circle $c$ ).

Then the vector function of the cyclical cycloidal surface $\Phi_{1}^{\prime}$ is

$$
\mathbf{P}_{1}^{\prime}(u, v)=\mathbf{r}_{1}(v)+\mathbf{c}_{1}^{\prime}(u) \cdot \mathbf{M}_{1}\left(m_{1} v\right), u \in\langle 0,2 \pi\rangle, v \in\langle 0,2 \pi\rangle,
$$

where $\mathbf{r}_{1}(v)$ is vector function of the cycloidal curve $c_{1}$ expressed in equations $(8), \mathbf{M}_{1}\left(m_{1} v\right)$ is the transformation matrix (Eq.12) and $\mathbf{c}_{1}^{\prime}(u)=\left(r_{1}^{\prime} \cos u, r_{1}^{\prime} \sin u, 0,1\right), u \in\langle 0,2 \pi\rangle$ is the vector function of the circle $c_{1}^{\prime}$ moving along the curve $c_{1}$ and lying in its normal plane. [4], [6]

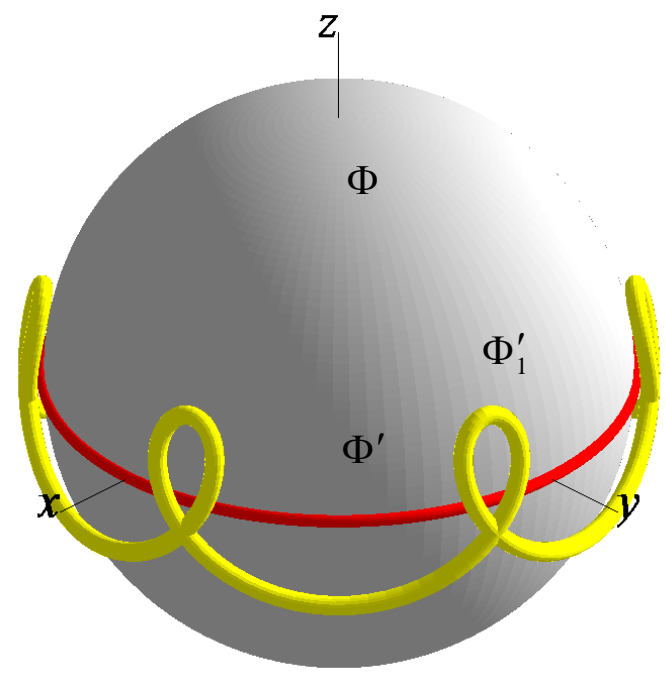

Figure 2

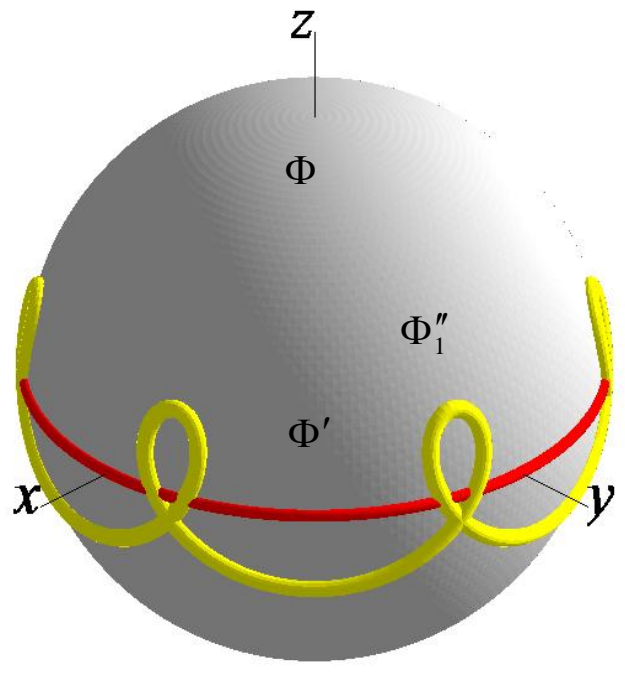

Figure 3

In Fig.2 are displayed spherical surface $\Phi$, cyclical surface $\Phi^{\prime}$ and cycloidal cyclical surface $\Phi_{1}^{\prime}$, determined by parameters $r=15, m_{1}=6, d_{1}=5, r^{\prime}=0.35, \operatorname{sgn}_{1}=-1, r_{1}^{\prime}=0.5$.

If we project every point $\mathrm{X}_{1}\left(x_{1}, z_{1}, z_{1}, 1\right)$ of the cycloidal curve $c_{1}$ on the spherical surface $\Phi$ by using the central projection with centre of the spherical surface, then we obtain points $\mathrm{X}_{1}^{\prime}\left(x_{1}^{\prime}, y_{1}^{\prime}, z_{1}^{\prime}, 1\right)$ which create the cycloidal curve $s_{1}^{\prime}$ on the spherical surface $\Phi$. Then transformation betveen coordinates is determined by the equations

$$
x_{1}^{\prime}=\frac{r x_{1}}{\sqrt{x_{1}^{2}+y_{1}^{2}+z_{1}^{2}}}, y_{1}^{\prime}=\frac{r y_{1}}{\sqrt{x_{1}^{2}+y_{1}^{2}+z_{1}^{2}}}, z_{1}^{\prime}=\frac{r z_{1}}{\sqrt{x_{1}^{2}+y_{1}^{2}+z_{1}^{2}}} .
$$

Then cycloidal cyclical surface $\Phi_{1}^{\prime \prime}$ on the spherical surface $\Phi$ is created by the movement of the circle $c_{1}^{\prime}=\left(O^{\prime \prime}, r_{1}^{\prime}\right)$ along the cycloidal curve $c_{1}^{\prime}$ and lying in the normal plane determined by the principal normal $n^{\prime}$ and the binormal $b^{\prime}$ of the curve $c_{1}^{\prime}$ at the point $O^{\prime \prime}$. 
In Fig.3 are displayed spherical surface $\Phi$, cyclical surface $\Phi^{\prime}$ and cycloidal cyclical surface $\Phi_{1}^{\prime \prime}$ determined by the same parameters as in the Fig.2.

In Fig.4 are displayed spherical surface $\Phi$, cyclical surface $\Phi^{\prime}$ and cycloidal cyclical surface $\Phi_{1}^{\prime \prime}$, determined by the same parameters as in the Fig.2., except $\operatorname{sgn}_{1}=+1$.

In Fig.5 are displayed spherical surface $\Phi$, cyclical surface $\Phi^{\prime}$ and cycloidal cyclical surface $\Phi_{1}^{\prime \prime}$, with same parameters as in the Fig.4., except $m_{1}=8$.

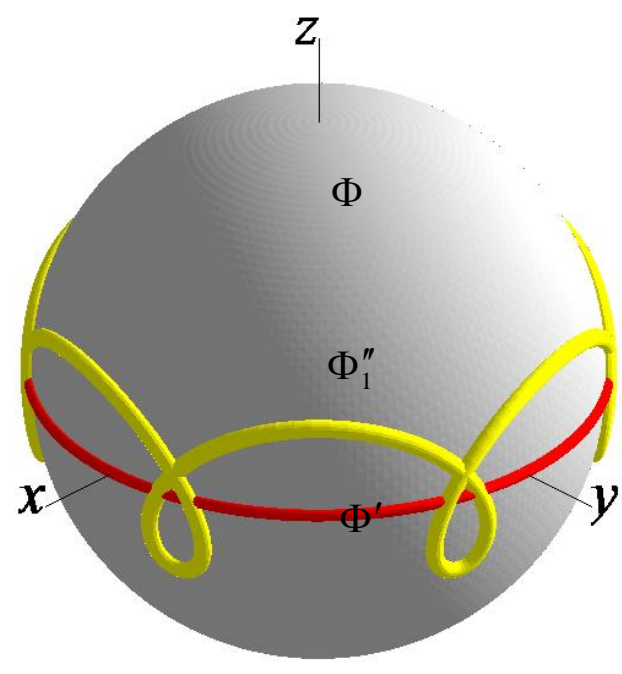

Figure 4

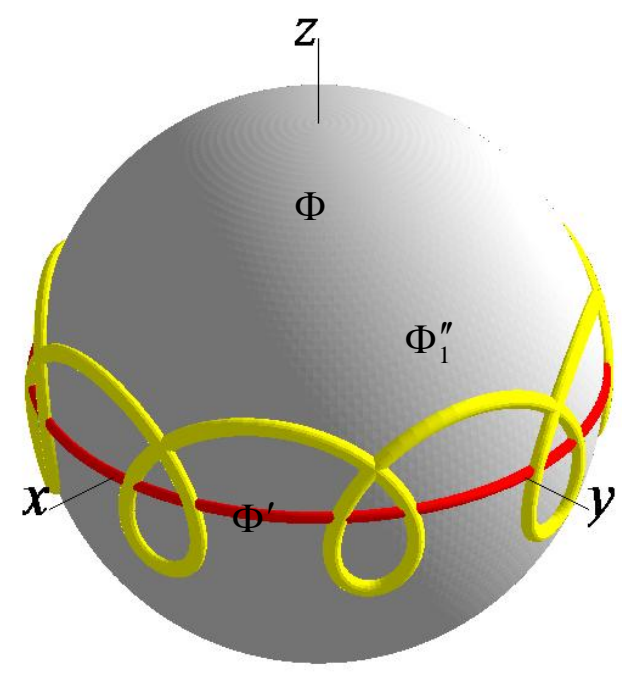

Figure 5

\section{Creating of the binormal cycloidal curve $c_{2}$ of the curve $c_{1}$}

Point $\mathrm{P}_{2}\left(d_{2}, 0,0,1\right)$ in the coordinate system $\left(0^{\prime \prime}, n^{\prime}, b^{\prime}, t^{\prime}\right), O^{\prime \prime} \in c_{1}$ rotates about the binormal $b^{\prime}$ of the cycloidal curve $c_{1}$ with angular velocity $m_{2} v$ and orientation determined by the parameter $\operatorname{sgn}_{2}$ and creates the cycloidal curve $c_{2}$ with vector function

$$
\mathbf{r}_{2}(v)=\left(x_{2}, y_{2}, z_{2}, 1\right)=\left(x_{1}+x_{01} n_{x}^{\prime}+z_{01} t_{x}^{\prime}, y_{1}+x_{01} n_{y}^{\prime}+z_{01} t_{y}^{\prime}, z_{1}+x_{01} n_{z}^{\prime}+z_{01} t_{z}^{\prime}, 1\right),
$$

where parameter $v \in\langle 0,2 \pi\rangle$ and

$$
x_{01}=d_{2} \cos m_{2} v, z_{01}=\operatorname{sgn}_{2} d_{2} \sin m_{2} v .
$$

Cycloidal cyclical surface $\Phi_{2}^{\prime}$ is created by the movement of the circle $c_{2}^{\prime}=\left(O^{\prime \prime \prime}, r_{2}^{\prime}\right)$ along the cycloidal curve $c_{2}$ and lying in the normal plane determined by the principal normal $n^{\prime \prime}$ and the binormal $b^{\prime \prime}$ of the curve $c_{2}$ at the point $0^{\prime \prime \prime}$. 
Let $\left(O^{\prime \prime \prime}, n^{\prime \prime}, b^{\prime \prime}, t^{\prime \prime}\right)$ be the Frenet-Serret moving trihedron of the cycloidal curve $c_{2}$, where $n^{\prime \prime}$ is the principle normal, $b^{\prime \prime}$ is binormal and $t^{\prime \prime}$ is tangent of thecurve $c_{2}$ at the point $O^{\prime \prime \prime} \in c_{2}$ with the unit vectors

$$
\begin{gathered}
\mathbf{t}^{\prime \prime}(v)=\left(t_{x}^{\prime \prime}, t_{y}^{\prime \prime}, t_{z}^{\prime \prime}\right)=\frac{\mathbf{r}_{2}(v)^{\prime}}{\left|\mathbf{r}_{2}(v)^{\prime}\right|}, \\
\mathbf{b}^{\prime \prime}(v)=\left(b_{x}^{\prime \prime}, b_{y}^{\prime \prime}, b_{z}^{\prime \prime}\right)=\frac{\mathbf{r}_{2}(v)^{\prime} \times \mathbf{r}_{2}(v)^{\prime \prime}}{\left|\mathbf{r}_{2}(v)^{\prime} \times \mathbf{r}_{2}(v)^{\prime \prime}\right|}, \\
\mathbf{n}^{\prime \prime}(v)=\left(n_{x}^{\prime \prime}, n_{y}^{\prime \prime}, n_{z}^{\prime \prime}\right)=\mathbf{b}^{\prime \prime}(v) \times \mathbf{t}^{\prime \prime}(v) .
\end{gathered}
$$

Then matrix

$$
\mathbf{M}_{2}(v)=\left(\begin{array}{cccc}
n_{x}^{\prime \prime}(v) & n_{y}^{\prime \prime}(v) & n_{z}^{\prime \prime}(v) & 0 \\
b_{x}^{\prime \prime}(v) & b_{y}^{\prime \prime}(v) & b_{z}^{\prime \prime}(v) & 0 \\
t_{x}^{\prime \prime}(v) & t_{y}^{\prime \prime}(v) & t_{z}^{\prime \prime}(v) & 0 \\
0 & 0 & 0 & 1
\end{array}\right)
$$

represents the transformation of the coordinate system $\left(0^{\prime \prime \prime}, n^{\prime \prime}, b^{\prime \prime}, t^{\prime \prime}\right)$ (the moving trihedron of the curve $c_{2}$ ) into the coordinate system $\left(0^{\prime \prime}, n^{\prime}, b^{\prime}, t^{\prime}\right)$ (the moving trihedron of the curve $c_{1}$ ). $[8],[9]$

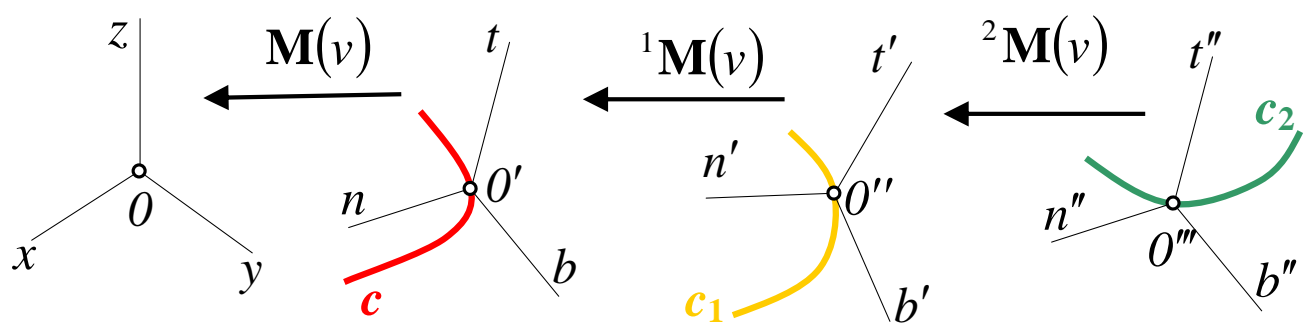

Figure 6: Transformations of the coordinate systems $\left(O^{\prime \prime \prime}, n^{\prime \prime}, b^{\prime \prime}, t^{\prime \prime}\right) \rightarrow\left(O^{\prime \prime}, n^{\prime}, b^{\prime}, t^{\prime}\right) \rightarrow\left(O^{\prime}, n, b, t\right) \rightarrow(0, x, y, z)$

In Fig. 6 are displayed transformations of the coordinate system $\left(O^{\prime \prime \prime}, n^{\prime \prime}, b^{\prime \prime}, t^{\prime \prime}\right)$ (the moving trihedron of the cycloidal curve $\left.c_{2}\right)$ to the coordinate system $\left(O^{\prime \prime}, n^{\prime}, b^{\prime}, t^{\prime}\right)$ (the moving trihedron of the cycloidal curve $\left.c_{1}\right),\left(O^{\prime \prime}, n^{\prime}, b^{\prime}, t^{\prime}\right)$ to the $\left(O^{\prime}, n, b, t\right)$ (the moving trihedron of the circle $c)$ and $\left(O^{\prime}, n, b, t\right)$ to the coordinate system $(o, x, y, z)$. 
The vector function of the cyclical cycloidal surface $\Phi_{2}^{\prime}$ is

$$
\mathbf{P}_{2}^{\prime}(u, v)=\mathbf{r}_{2}(v)+\mathbf{c}_{2}^{\prime}(u) \cdot \mathbf{M}_{2}\left(m_{2} v\right), u \in\langle 0,2 \pi\rangle, v \in\langle 0,2 \pi\rangle,
$$

where $\mathbf{r}_{2}(v)$ is vector function of the cycloidal curve $c_{2}$ expressed in equations (15), $\mathbf{M}_{2}\left(m_{2} v\right)$ is the transformation matrix (Eq.20) and $\mathbf{c}_{2}^{\prime}(u)=\left(r_{2}^{\prime} \cos u, r_{2}^{\prime} \sin u, 0,1\right), u \in\langle 0,2 \pi\rangle$ is the vector function of the circle $c_{2}^{\prime}$ moving along the curve $c_{2}$ and lying in its normal plane.

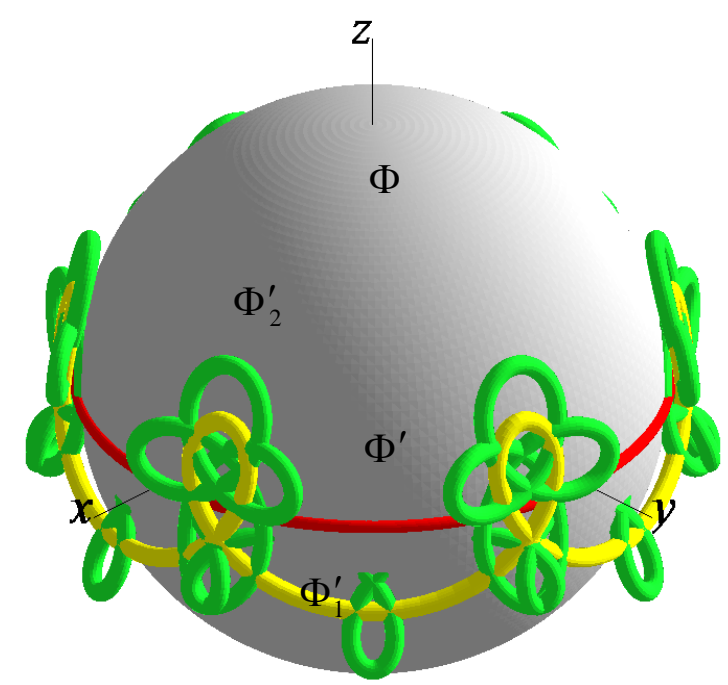

Figure 7

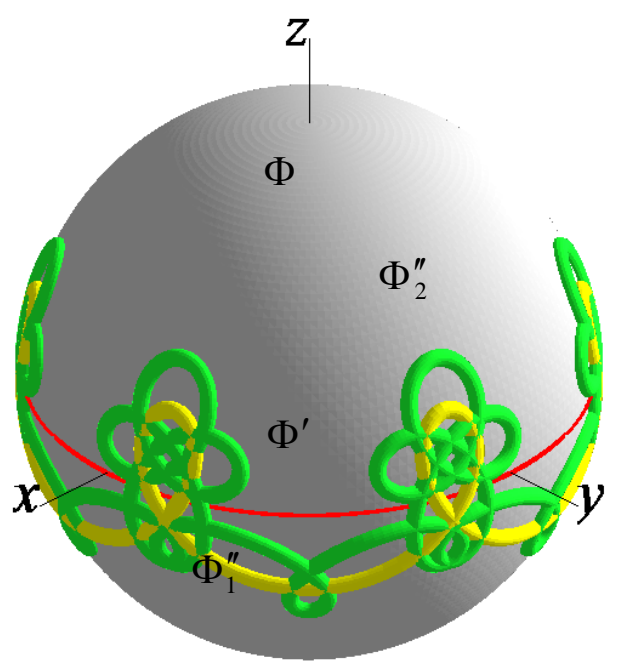

Figure 8

In Fig.7 are displayed spherical surface $\Phi$, cyclical surface $\Phi^{\prime}$, cycloidal cyclical surface $\Phi_{1}^{\prime}$ and cycloidal cyclical surface $\Phi_{2}^{\prime}$ determined by the parameters $r=15, m_{1}=6, d_{1}=5$, $r^{\prime}=0.35, \operatorname{sgn}_{1}=-1, r_{1}^{\prime}=0.5, m_{2}=6 m_{1}, d_{2}=3, \operatorname{sgn}_{2}=+1, r_{2}^{\prime}=0.5$.

If we project every point $\mathrm{X}_{2}\left(x_{2}, z_{2}, z_{2}, 1\right)$ of the cycloidal curve $c_{2}$ on the spherical surface $\Phi$ by using the central projection with centre of the spherical surface, then we obtain points $\mathrm{X}_{2}^{\prime}\left(x_{2}^{\prime}, y_{2}^{\prime}, z_{2}^{\prime}, 1\right)$ creating the cycloidal curve $s_{2}^{\prime}$ on the spherical surface $\Phi$. Then transformation betveen coordinates is determined by the equations

$$
x_{2}^{\prime}=\frac{r x_{2}}{\sqrt{x_{2}^{2}+y_{2}^{2}+z_{2}^{2}}}, y_{2}^{\prime}=\frac{r y_{2}}{\sqrt{x_{2}^{2}+y_{2}^{2}+z_{2}^{2}}}, z_{2}^{\prime}=\frac{r z_{2}}{\sqrt{x_{2}^{2}+y_{2}^{2}+z_{2}^{2}}} .
$$

Then cycloidal cyclical surface $\Phi_{2}^{\prime \prime}$ on the spherical surface $\Phi$ is created by moving circle $c_{2}^{\prime}=\left(O^{\prime \prime \prime}, r_{2}^{\prime}\right)$ along the cycloidal curve $c_{2}^{\prime}$ and lying in the normal plane determined by the principal normal and the binormal of the curve $c_{2}^{\prime}$ at the point $0^{\prime \prime \prime}$. 
In Fig. 8 are displayed spherical surface $\Phi$, cyclical surface $\Phi^{\prime}$, cycloidal cyclical surface $\Phi_{1}^{\prime \prime}$ and $\Phi_{2}^{\prime \prime}$ determined by the same parameters as in the Fig.7.

\section{Creating the ornaments on the spherical surface by using normal and binormal cycloidal cyclical surfaces}

We can create various ornaments on the spherical surface by the changing of the parameters of the cycloidal curves $c_{1}, c_{2}$ as a determining cuves of the cyclical cylindrical surfaces $\Phi_{1}^{\prime}$ and $\Phi_{2}^{\prime}$ and their projected surfaces $\Phi_{1}^{\prime \prime}$ and $\Phi_{2}^{\prime \prime}$ on the spherical surface $\Phi$.

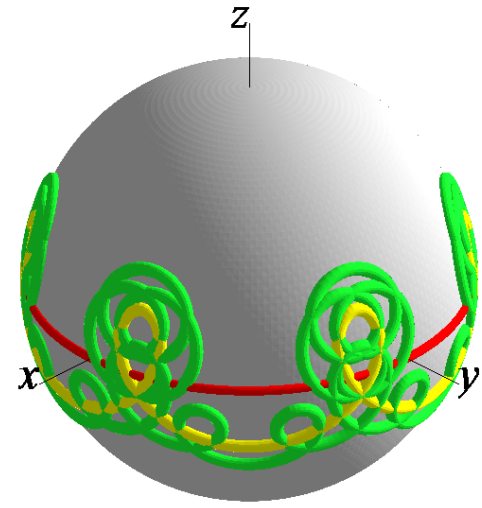

Figure 9

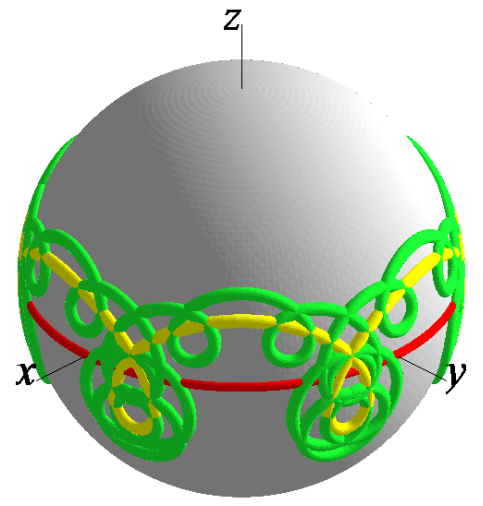

Figure 10

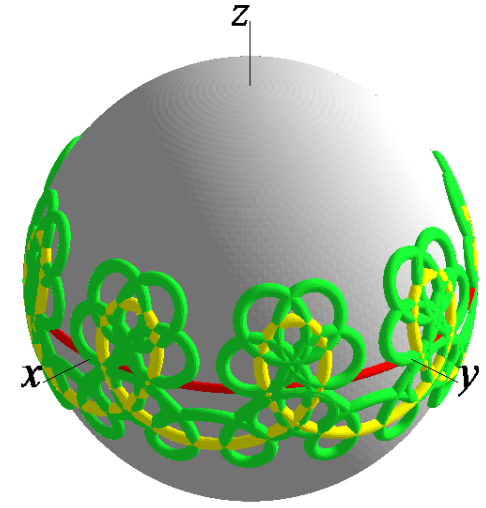

Figure 11

In Fig.9 are displayed spherical surface $\Phi$, cyclical surface $\Phi^{\prime}$, cycloidal cyclical surface $\Phi_{1}^{\prime \prime}$ and $\Phi_{2}^{\prime \prime}$, determined by the same parameters as in the Fig.7 except $\operatorname{sgn}_{1}=-1, \operatorname{sgn}_{2}=-1$, in Fig. 10 are changed parameters $\operatorname{sgn}_{1}=+1, \operatorname{sgn}_{2}=-1$ and in Fig.11 are changed parameters $m_{1}=8, m_{2}=8 m_{1}, \operatorname{sgn}_{1}=-1, \operatorname{sgn}_{2}=+1$.

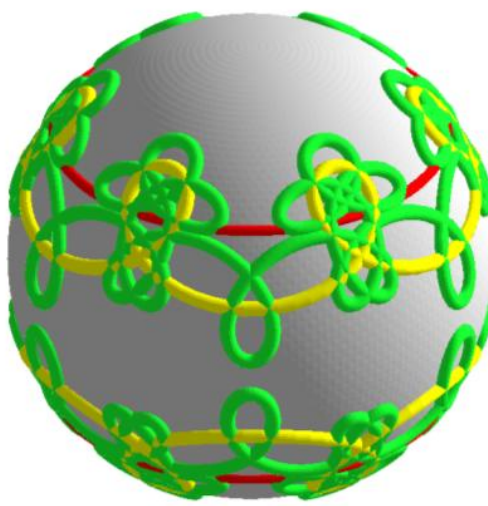

Figure 12

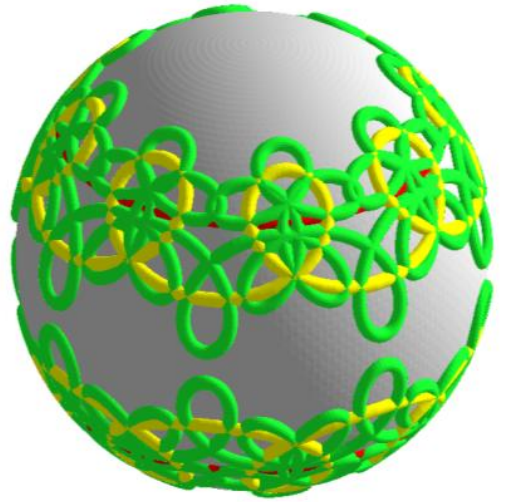

Figure 13

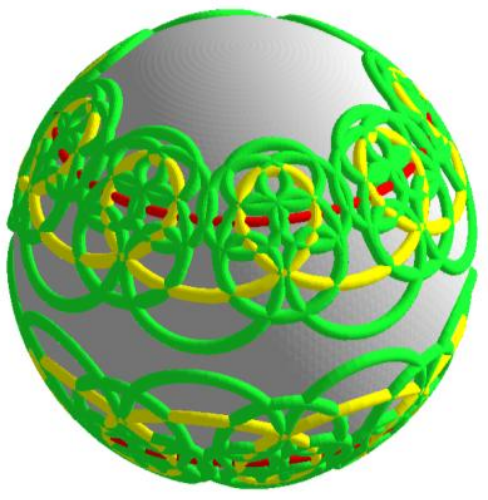

Figure 14 
In Fig.12 are displayed spherical surface $\Phi$, cyclical surfaces ${ }^{1} \Phi^{\prime},{ }^{2} \Phi^{\prime}$ created by using the circles ${ }^{1} c,{ }^{2} c$ determined by vector functions

$$
\begin{gathered}
{ }^{1} \mathbf{r}(v)=(r \cos \alpha \cos v, r \cos \alpha \sin v, r \sin \alpha, 1), v \in\langle 0,2 \pi\rangle, \\
{ }^{2} \mathbf{r}(v)=(r \cos \alpha \cos v, r \cos \alpha \sin v,-r \sin \alpha, 1), v \in\langle 0,2 \pi\rangle,
\end{gathered}
$$

cycloidal cyclical surfaces ${ }^{1} \Phi_{1}^{\prime \prime},{ }^{2} \Phi_{1}^{\prime \prime}$ and ${ }^{1} \Phi_{2}^{\prime \prime},{ }^{2} \Phi_{2}^{\prime \prime}$ determined by vector functions

$$
\begin{gathered}
{ }^{1} \mathbf{P}_{1}^{\prime}(u, v)={ }^{1} \mathbf{r}_{1}(v)+\mathbf{c}_{1}^{\prime}(u) .{ }^{1} \mathbf{M}_{1}\left(m_{1} v\right),{ }^{2} \mathbf{P}_{1}^{\prime}(u, v)={ }^{2} \mathbf{r}_{1}(v)+\mathbf{c}_{1}^{\prime}(u) .{ }^{2} \mathbf{M}_{1}\left(m_{1} v\right), \\
{ }^{1} \mathbf{P}_{2}^{\prime}(u, v)={ }^{1} \mathbf{r}_{2}(v)+\mathbf{c}_{2}^{\prime}(u) .{ }^{1} \mathbf{M}_{2}\left(m_{2} v\right),{ }^{2} \mathbf{P}_{2}^{\prime}(u, v)={ }^{2} \mathbf{r}_{2}(v)+\mathbf{c}_{2}^{\prime}(u) .{ }^{2} \mathbf{M}_{2}\left(m_{2} v\right),
\end{gathered}
$$

Determined by the parameters $r=15, m_{1}=6, d_{1}=5, \quad r^{\prime}=0.35, \quad \operatorname{sgn}_{1}=-1, \quad r_{1}^{\prime}=0.5$, $d_{2}=3, m_{2}=6 m_{1}, \operatorname{sgn}_{2}=+1, r_{2}^{\prime}=0.5, \alpha=\pi / 5$.

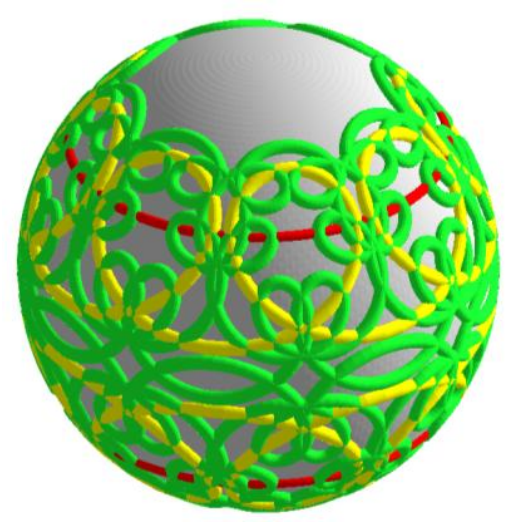

Figure 15

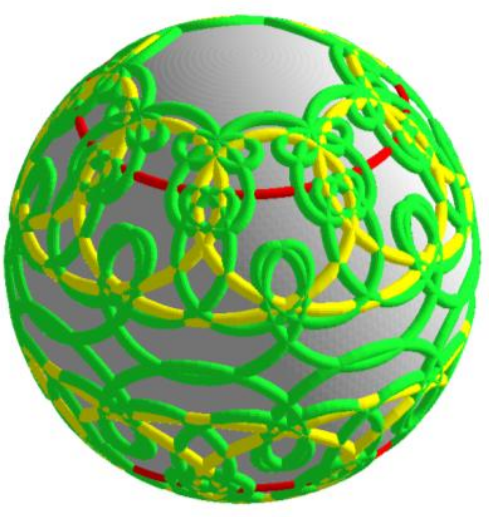

Figure 16

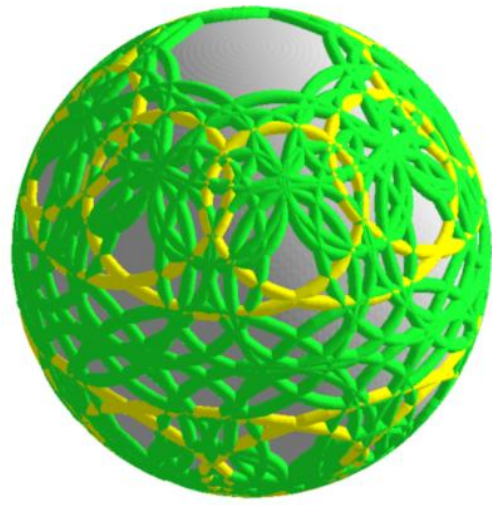

Figure 17

In Fig.14 is changed parameter $m_{1}=8$, in Fig. 15 is changed parameter $\operatorname{sgn}_{2}=-1$, in Fig 16 is changed parameter $d_{1}=7$, in Fig. 17 are changed parameters $d_{1}=8, \alpha=\pi / 4$, in Fig. 16 is change parameter $d_{2}=6$.

\section{Conclusion}

By using of the mathematical apparatus we can create spatial ornaments as an example of the utilization of mathematics in design. The design is also needed in addition purposeful and aesthetic.

The described method of the modeling of cyclical cycloidal surfaces on the spherical surface shows a simply way to modeling of different interesting ornaments on the spherical surface by changing of parameters. 
In this paper was used rotations about the coordinate axes of the moving trihedrons of the curves lying on the spherical surface.

\section{Acknowledgements}

This work was supported by VEGA 1/0321/12 „Theoretical and experimental analysis of adaptive cable and tensegrity systems under static and dynamic stress considering the effect of wind and seismic".

\section{References}

[1] Budinský,B., Kepr,B. (1970). Introduction to differential geometry with technical applications. SNTL-Publishers of technical literature, Praha.

[2] Granát, L., Sechovský, H. (19800. Computer graphics. SNTL-Publishers of technical literature, Praha.

[3] Olejníková,T. (2010). Two helical surfaces. Journal of civil engineering, Selected scientific papers. Vol. 5, (issue 1), pp.7-16.

[4] Olejníková,T. (20070 Composed Cyclical Surfaces. Transactions of the universities of Košice. Volume 3, pp.54-60.

[5] Olejníková,T. (2012). Rope of Cyclical Helical surfaces. Journal of civil engineering, Selected scientific papers. Vol. 7, (issue 2), pp.23-32. DOI: 10.2478/v10299-012-0003-4.

[6] Olejníková,T. (2013). Cyclical surfaces created by helix on general surface of revolution. Journal of civil engineering, Selected scientific papers. Vol. 8, (issue 2), pp.33-40. DOI: 10.2478/sspjce-2013-0016.

[7] Olejníková,T. (2013). Helical-one, two, three-revolutional cyclical surfaces. Global journal of science frontier research, Mathematics and decision sciences. Vol. 13. (issue 4), pp. 47-56.

[8] Študencová,Z., Zámožík,J., Szarková,D. (20120. Skrut-Art. G-Slovak journal of geometry and grafic. Vol. 9, (issue 17), pp. 41-52.

[9] Olejníková, T. (2014). Cyclical Surfaces Created by Helix on Torus. International Journal of Applied Mathematics. Vol. 2,(issue 6), p. 204-208. DOI: 10.11648/j.ajam.20140206.12. 